\title{
When microbiologists plunge into the ocean
}

Microbiologists stake their claim: assessing climate change involves new ways of studying the ocean's microbes.

\author{
Vivien Marx
}

M icrobiology wants in. No longer should microbes and microbial processes be left out of climate change assessments, state 33 researchers from nine countries in their consensus statement ${ }^{1}$, "Scientists' warning to humanity: microorganisms and climate change." There's a "need to act," the authors write. By underappreciating the importance of microbial processes both on land and in the oceans, "we fundamentally limit our understanding of Earth's biosphere and response to climate change and thus jeopardize efforts to create an environmentally sustainable future." The consensus statement is still collecting signatories, which at press time numbered 1,000 , including individual scientists and 70 organizations. This warning is motivated by others, such as those from the Alliance of World Scientists and the Union of Concerned Scientists. Rick Cavicchioli, from Australia's University of New South Wales and first author of the consensus statement, wanted to add the voice of microbiologists. The United Nations Decade of Ocean Science for Sustainable Development begins in 2021, which will add urgency to ocean research. To study marine microorganisms such as bacteria and archaea, eukaryotic plankton, viruses, and fungi, researchers develop and use genomics and metagenomics tools, diversify sample collection, and build models and new types of ocean observatories. They want to dissect how microbes affect climate, how climate affects microbes and, potentially, how microbes might mitigate climate change. This new and necessary focus of the field is "global change microbiology," says Antje Boetius, who directs the Alfred Wegener Institute Helmholtz Centre for Polar and Marine Research, in Bremerhaven, Germany, and who coauthored the consensus statement. "We totally agree with this call to action," says environmental microbiologist Lucie Zinger from the Institut de Biologie de l'École Normale Supérieure (IBENS) in Paris. Microbes drive biogeochemical cycles on Earth. It's timely to emphasize the critical need to incorporate the often-neglected microbes into our thinking about climate change effects, says microbiome researcher Shinichi

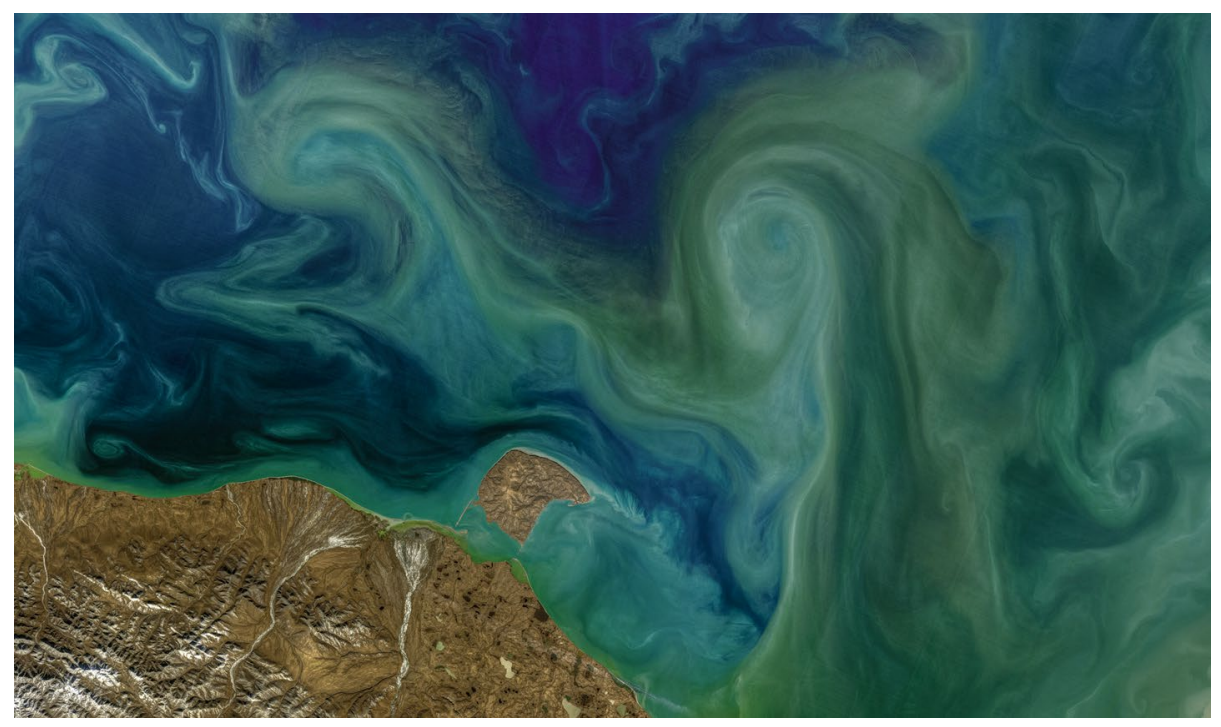

Scientists seek more detail on the interaction between the ocean's microbes and climate. Here, the Landsat 8 satellite shows phytoplankton swirls in the sea north of Canada's Yukon Territory. Credit: NASA Goddard Space Flight Center OceanColor Web

Sunagawa from ETH Zurich. "Consequently, I signed the statement without any hesitation," he says. "We may be closing gaps in our knowledge about ocean microbial biodiversity, but our knowledge about global trends of changes of this diversity are indeed still very fragmentary." Many factors shape changes in abundances and composition of oceanic microbial communities, with hardto-anticipate effects. Deeper understanding of this dynamic takes commitment to applying experimental methods in a consistent and long-term way, says Boetius, highlighting the work of Charles Keeling. "Where would we be today without this man?" she says. Starting with the question 'can I see plants and microbes breathe?' he devised a way to systematically measure carbon dioxide in the air. Measurements started in 1958 and continue today. They have revealed seasonal patterns and rising $\mathrm{CO}_{2}$ levels due to human activities, which is called the Keeling curve in his honor. There is no Keeling curve for the oceans. To assess how microbial processes change might take curves for much of the periodic table, says Boetius. Much $\mathrm{CO}_{2}$ from burning fossil fuel is absorbed in the oceans. Increases lead to acidification, which can damage marine ecosystems such as coral reefs, note Ken Caldeira from the Carnegie Institution for Science and colleagues ${ }^{2}$. When ocean chemical conditions improve, these 'calcifiers' may recover, says Caldeira. In a few years, the National Aeronautics and Space Administration will launch PACE, the Plankton Aerosol, Cloud, Ocean Ecosystem. According to NASA, the satellite will lack the 'blind spots' of previous approaches. For example, its hyperspectral imaging system will cover 340 to 890 nanometers and resolve greater detail about phytoplankton communities.

\section{Who's where}

To assess changes to microbial communities, some research groups study microbial taxa such as the bacterial clade SAR11, also known as Pelagibacteraceae. SAR11 might comprise as much as one-third of all marine bacteria. They're found from pole to pole, from ocean surface to seafloor, and mainly inhabit tropical to temperate waters. Using $16 \mathrm{~S}$ rRNA genes as well as whole-genome sequencing, Cavicchioli and colleagues have explored global SAR11 distribution and found that temperature and latitude shape SAR11 
phylotypes, which is classification based on gene similarity. The team pored over 128 marine metagenomes based on samples from surface waters around the world and 37 from Antarctic waters. The team included data from J. Craig Venter's Global Ocean Sampling Expeditions. To consider phylotype distribution and changing ocean temperatures, they integrated findings from the Intergovernmental Panel on Climate Change reports. It appears, says Cavicchioli, that as the polar ocean warms, SAR11 phylotypes might turn over and be replaced by the incursion of phylotypes from warmer waters. SAR11's abundance enables such oceans-wide analysis. It will take more data to study less abundant microbes, he says. Characterization can be tough because many marine microbes won't grow in the lab. Culture-independent methods, such as community shotgun sequencing or targeted gene surveys, can help make communitylevel statements, he says, such as about metabolic traits. That's true for studying microbes in the oceans, on land and in the human gut. Single-cell genomics helps to better interrogate metagenome data, he says. The data can be assigned to uncultured taxonomic groups to give labs clues regarding these microbes' needs so they can be coaxed to grow in the lab. This connects single-cell genomics and research on oceanic microbial dynamics. Natural historian David Attenborough practices discovery science when he overturns a rock to see what life forms are there, says Cavicchioli. The ocean can be such a 'rock' to which modern tools such as metagenomic analysis are applied to study microbial communities. Alternatively, an experiment can start with a hypothesis. Using both approaches, he and his team are redefining views of the phylum Nanohaloarchaeota, salt-adapted archaea that live in cold, salty Antarctic lakes ${ }^{3}$. They were thought to be free-living microbes and not culturable in the lab. Neither seems to be true. These microbes can't synthesize lipids, amino acids and nucleotides and must grow in symbiosis with a host, a haloarchaeon. As such, this a model system for studying archaeal symbiosis. To gain this insight, the team used many methods: field work and microbial culture, genomics and proteomics, flow cytometry, fluorescence and electron microscopy.

\section{Global ocean metatranscriptomics}

Other groups assess changes to the metatranscriptome, which is the readout of messenger RNA transcripts from microbial communities. Sunagawa and colleagues sought to capture such community-level variation on a global scale ${ }^{4}$. They studied turnover and change in composition -

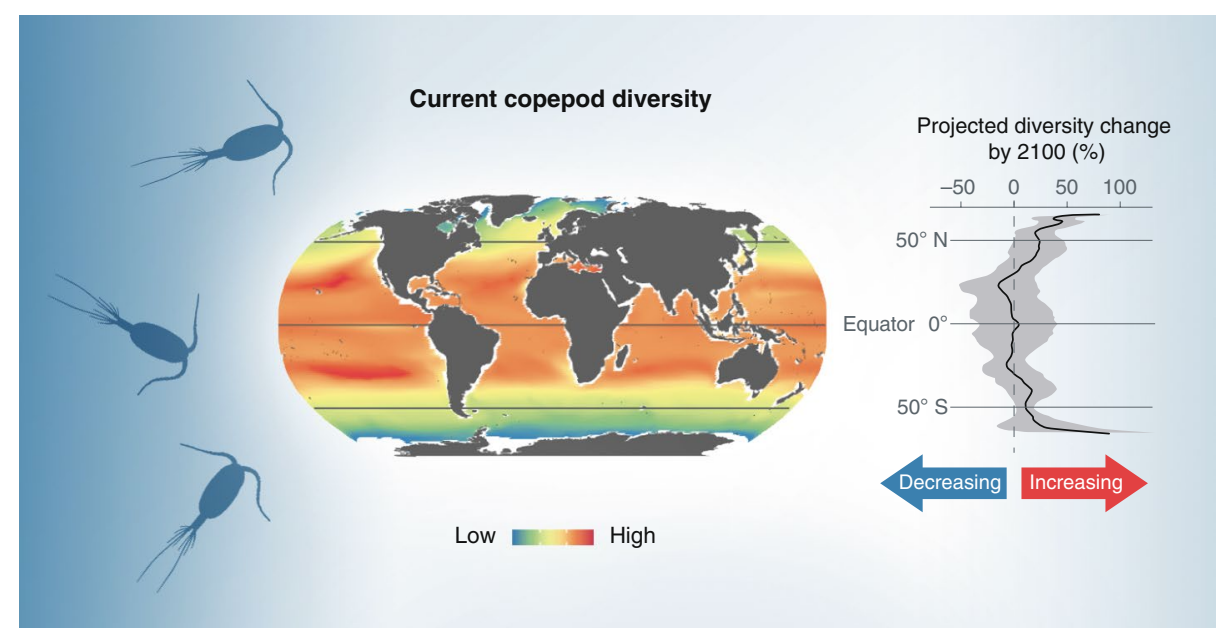

Researchers at IBENS study global trends in marine plankton. As the sea surface temperature changes, so will plankton diversity. Credit: L. Zinger, C. Bowler, F. Ibarbalz, F. Lombard, IBENS and CNRS; J. Wolfe, Phylopic; K. Frischkorn, B. Langenbrunner / Springer Nature

that's the relative abundance of organisms and their expressed genes - to gauge aspects such as microbial influence in food webs, ocean biogeochemistry and possible responses to a changing climate. They looked at plankton, a diverse group of organisms that includes the abundant SAR11 and many others, including the photosynthetic Procholorococcus. Temperature turned out to be a major determinant of microbial composition. The team used data from Tara Oceans, a global sampling expedition from 2009 to 2013. Samples from 126 ocean sites were collected at depths between five meters and one kilometer. From a total of 557 samples, they obtained 370 metagenomes and 187 metatranscriptomes. They assessed microbial presence, molecular activity and microbial function, by, for example, looking at genes involved in photosynthesis or nutrient cycling. For profiling taxonomic composition with high-throughput sequence data of $16 \mathrm{~S}$ and $18 \mathrm{~S}$ rRNA gene fragments, the team used existing data resources. But for mapping metagenomes and metatranscriptomes they had to build a reference catalog of 47 million genes. In polar waters, says Sunagawa, the team found that organismal composition shaped the transcript pool more than gene expression did. In non-polar waters, however, changes in gene expression drove more variation in the transcript pool than community turnover. Given changing water surface temperatures in the Arctic Ocean, this hints at changes to come. Also working with Tara Oceans data, IBENS researchers Zinger and Chris Bowler and colleagues studied global trends in diversity and spatial patterns of all types of marine plankton, from viruses to microbes to zooplankton, from both Northern and Southern Hemispheres ${ }^{5}$. The team compiled taxonomic information about sequenced samples and used tools such as the watertight ZooScan imaging system to distinguish photosynthetic from non-photosynthetic plankton, which have different roles in the marine food web. To see how changes ocean $\mathrm{pH}$ or temperature might affect plankton and the biogeochemical cycles and food webs they sustain, they looked at latitudinal diversity gradients, according to which species diversity is highest at the equator and lessens toward the poles. It has not been well studied for marine plankton, says Zinger, and before their work global-scale knowledge about plankton, especially related to latitudinal gradients, was fragmentary and contradictory. Temperature has been thought to shape plankton diversity, and the team established that it does. Plankton diversity is lower in cooler oceans near the poles. The appearance of certain photosynthetic bacteria and copepods in the Arctic Ocean indicates some ongoing 'tropicalization' in this region. Climate models usually account for plankton as two coarse components: the phytoplankton and the zooplankton. Zinger says that these "do not reflect at all the complexity of the planktonic food-web, which includes all domains of life." Such models leave much out, such as viruses and most non-photosynthetic microbes, bacteria, archaea or protists that recycle organic matter produced in the ocean. Existing research indicates how climate change will affect marine food webs in the seas of the Northern Hemisphere, but "we still have strong uncertainties 
on the actual nature and magnitude of these changes," she says. The latitudinal diversity gradient is "typically the kind of mythical question any ecologist would like to address," says Zinger. Exceptions exist for the phenomenon of greater diversity at the equator than the poles, but it's pervasive. Science cannot explain this pattern, she says. What fascinates him, says Bowler, is that as dynamic as the ocean and its complex circulation patterns are on a global scale, plankton communities structure themselves into definable patterns. "We now have to understand the laws that govern this," he says. Temperature is certainly a major factor that shapes oceanic microbial diversity, he says, as do microbial composition, latitudinal diversity gradients and mechanisms of community transcriptome changes. Additional factors include deoxygenation, acidification, ocean pollution and long-term developments.

\section{Floating for data}

Capturing genomic information is still expensive, laborious and slow, says Sunagawa, compared to, for example, sensor-based data capture. Nearly 4,000 sensor-equipped, battery-powered Argo floats are deployed around the world to record temperature and salinity. These mini-observatories are run by teams in 30 countries. For around four years, a float moves autonomously up and down the water column, from the surface down to $2,000 \mathrm{~m}$. The float's density matches the density of the seawater depth where it is set to drift. Every ten days, the float rises to the surface by pumping oil from an internal chamber to an expandable external one to change its density. At the surface, the float sends data via satellite to scientists. Then the float

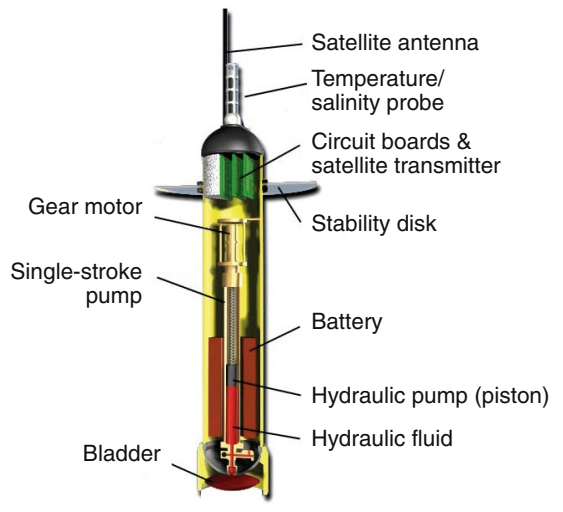

Globally, around 4,000 Argo floats record ocean temperature and salinity. They periodically rise to the surface and send data via satellite to researchers. Credit: Argo, www.argo.ucsd.edu; M. McClune, Scripps Institution of Oceanography sinks back to its depth. "In some future, methods for automated in-situ genomic data collection and transfer may become available, similar to Argo floats," says Sunagawa. That would give labs data about ocean life with greater spatial and temporal resolution. For an Argo float to do in situ -omics analysis, it would need "to extract nucleic acids and do DNA sequencing in a corrosive saltwater environment and to transmit the huge amounts of data via satellites," says Bowler. "It's perhaps 10 years away." But just because researchers can sequence all the genes in the ocean "doesn't mean that we should." To grow information about critical processes cost-efficiently and simply, in his view, researchers will want to define key processes and identify marker genes to monitor them. That might take a few years or decades to achieve, he says. To disentangle complex processes, work in the lab will include single-cell genomics and epigenetics analysis, and then "it's essential to go back to the field to evaluate true importance," he says. A technique with promise, in Sunagawa's view, is automated imaging combined with machine learning to classify and quantify larger organisms not only at ocean observatories but in the open ocean. Labs will also want to explore how to extrapolate from snapshots of the ocean data, he says, which is "what we will have to do in order to model, predict and perhaps modulate changes in the future." Technology keeps improving; instruments get smaller, cheaper and can run more autonomously, says Bowler. "But the biggest issue is to raise awareness about the importance of monitoring the biology of the ocean because the majority of oceanographers and climate scientists still consider that it's all about chemistry and physics, and that biology is not a major factor."

\section{Taking down seawalls}

"I do believe the interdisciplinary walls are currently very high, " says Sunagawa. "To bring them down, researchers will need to step out of our comfort zone," he says. Targeted incentives and funding schemes will help to promote cross-disciplinary efforts between physical oceanographers, climate modelers and ocean microbial ecologists to better address planetary-scale questions. Boetius says that her field of deep-sea research melds biogeochemistry and ecology. As a relatively small field, it involves thinking and working across disciplinary boundaries, developing methods and technology collaboratively and sharing them. In physical oceanography or astronomy, groups build global observatory infrastructure, "but in biology this is very hard." She often has discussions with biologists to start teasing out details of the "perfect biological ocean observatory" or the "perfect microbiome observatory." She and her colleagues studied a deep-sea microbiome that sits atop a methaneemitting volcano ${ }^{6}$. They sampled the mud, also right after the volcano erupted, and compared those microbial communities to others. They used $16 \mathrm{~S}$ rRNA sequence and metagenomic analysis to assess the ecosystem, its metabolic potential and the bacterial and archaeal gene families present. Systems questions take standardized and calibrated biological methods ${ }^{7}$. And they require that "you go beyond your own discipline and understand better cross-cutting questions and cross-cutting problems," she says. She and her teams have built and deployed the underwater observatory FRAM, or Frontiers in Arctic Marine Monitoring, to assess how climate change propagates through the deep sea. It has spent the past year in the Arctic deep sea to make physical oceanographic measurements, as well as collect data on deep sea animals and microbes. They have just pulled equipment out of the water and are poring over the data. "It's incredible," she says of her first impressions about data from an area where ice hinders satellite data collection. As part of FRAM, they are collaborating with the US Department of Energy's Joint Genome Institute in a group that includes biogeochemists and microbiologists. One explicit goal is to assess changes in the Arctic microbial ecosystem. They developed standardized methods, says Boetius, from the sampling protocols to the sequencing and metatranscriptomic analysis methods. The systems' modules include a microbial observatory that moves along the water column, collects samples, filters and performs in situ molecular analysis; a bio-optical platform that tallies organic matter to assess mass fluxes in the ocean and makes temperature and chlorophyll measurements; and instruments that take high-resolution photos of organisms between $0.2 \mathrm{~mm}$ and $2 \mathrm{~cm}$ in size. A benthic observatory sits on the seafloor and measures flux and biogeochemical parameters. Physical oceanographer Jennifer MacKinnon at the Scripps Institution of Oceanography says that studying factors such as turbulence or ocean circulation doesn't bring her into regular contact with marine microbiologists. She does not yet fully appreciate the role of microbial communities in climate change but, to begin to address that, she has a collaboration with a microbiologist in the works. "For years there has been a disparity between the types of measurements that, for example, physical and biological oceanographers could make, 


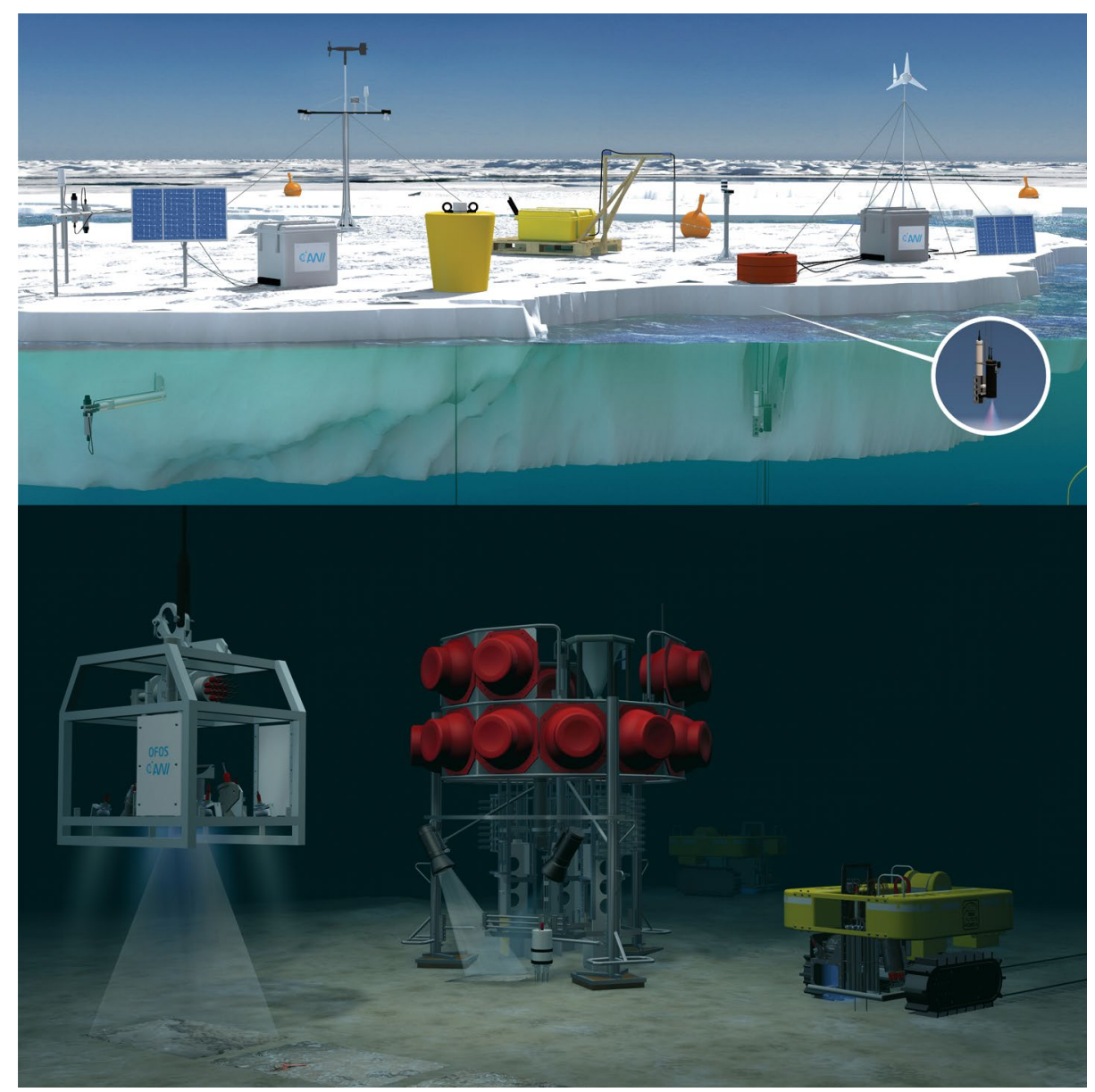

Frontiers in Arctic Marine Monitoring (FRAM) is a modular system for assessing the effects of climate change on the ocean. It includes ice-tethered instruments, a seafloor system and remote-controlled instruments that also collect microbial data. Credit: Alfred Wegener Institute / FRAM / S. Lüdeling

which limited the productive ground for interdisciplinary work," she says. It's been a hot topic in physical oceanography research of late to focus on sub-mesoscale dynamics, says MacKinnon. Mesoscale might involve eddies in the ocean, such as Gulf Stream rings around one hundred kilometers wide. That's how coarse the measurements and resolution of numerical measurements have been. But advances in ocean observational technology, methods and higher-resolution models are enabling the study of ocean dynamics on smaller scales: lateral scales of tens of kilometers down to mere meters horizontally, and everything in between. "It turns out there is a whole host of fascinating dynamics in this regime - sharp fronts, instabilities, swirls upon swirls," she says. "Which are not only lovely, but seem to matter for things setting the lateral and vertical distribution of heat and freshwater in the ocean." In satellite images of chlorophyll levels in the oceans, the swirls reflect sub-mesoscale ocean currents and instabilities and likely have large implications for ocean biology from the microbial level on up, she says, "but we really don't understand how." Only recently have higher-resolution biological measurements become possible. As research vessels move, they can now take in a continuous stream of water and quickly perform in situ genetic analysis of microbial communities in that water. To some extent, it can lower interdisciplinary walls to have technology that allows coincident highresolution sampling of relevant physical and biological quantities, she says. Funding mechanisms need to be tuned for such cross-disciplinary work. At the US National Science Foundation, for example, physical and biological oceanographers review proposals for their respective disciplines, and both panels review interdisciplinary proposals, says MacKinnon. "Word on the street is that these are very hard to get funded because it's near impossible to meet the standards of both groups," she says. For the physical oceanography reviewers, projects will lack "hard core" physics. Similar issues will be true for biologists looking at proposals. One option might be targeted federal funding calls geared toward interdisciplinary, focused work. Global climate models, or even regional models, "will not in our lifetimes resolve all the small-scale swirly stuff," she says - the factors that ultimately stir the ocean and mix heat, dissolve greenhouse gasses and distribute biologically essential nutrients in the ocean. They need to be well-represented in climate models, which have to be based on the "right" science. "If we don't know how those processes really work, it's hard to represent them correctly," she says. MacKinnon led a Climate Process and Modeling Team (CPT) project funded primarily by the National Science Foundation to develop better parameterizations of ocean turbulence for use in global climate models ${ }^{8}$, to capture events at a finer scale than the typical horizontal grid spacing of around 100 kilometers. Lab and field measurements show a role for internal gravity waves that propagate through the stratified interior of the oceans. Many factors, including ocean currents and sea surface winds, shape how these waves interact. Their turbulent mixing moves nutrients and dissolved $\mathrm{CO}_{2}$. MacKinnon and her team built an online repository of ocean mixing analysis tools and compared turbulence profilers, which can reach quite different quantitative results. This project only involves the physical side of oceanography, says MacKinnon, but "having an equivalent CPT for biological things would be great," she says.

CPTs by other groups consider processes such as boundary layer dynamics or how to best include sea ice in climate models. At Scripps and elsewhere, colleagues are building new types of instruments to make both physical and biological measures in the oceans. "My two cents is that making those measurements coincident with equally high-resolution biological measurements will be one of the hottest topics for the decade to come."

\section{Vivien Marx \\ Technology editor for Nature Methods. \\ e-mail:v.marx@us.nature.com}

Published online: 27 January 2020 https://doi.org/10.1038/s41592-020-0736-9

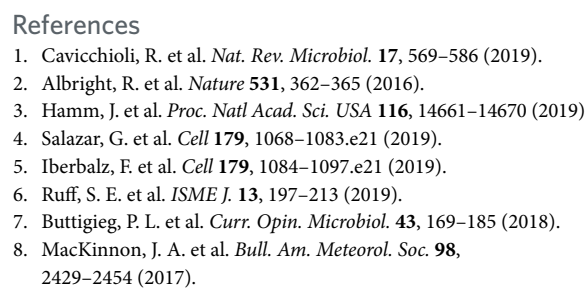

2. Albright, R. et al. Nature 531, 362-365 (2016)

14661-14670 (2019).

6. Ruff, S. E. et al. ISME J. 13, 197-213 (2019).

. 2429-2454 (2017) 